\title{
Inactividad Física y Ausentismo en el Ámbito Laboral
}

\author{
Sedentariness and absenteeism in the work setting \\ Elkin Martínez-López y Juan F. Saldarriaga-Franco \\ Facultad Nacional de Salud Pública. Universidad de Antioquia. Medellín, Colombia. elkmartz@guajiros.udea.edu.co, \\ juanfs@guajiros.udea.edu.co
}

Recibido 16 Junio 2007/Enviado para Modificación 29 Diciembre 2007/Aceptado 28 Marzo 2008

\section{RESUMEN}

Objetivo Explorar el comportamiento del ausentismo laboral y su relación con la inactividad física en una comunidad institucional.

Métodos Se comparan la frecuencia, duración, costo y causas de la incapacidad en relación con el sexo, la edad, y la práctica regular de actividad física. Se estudia el riesgo relativo $(\mathrm{RR})$ y se construyen intervalos de confianza al $95 \%$ para cada estimación.

Resultados El ausentismo laboral está asociado con el sedentarismo, RR 2,17 (IC95 \% 1,72-2,73). Las enfermedades respiratorias, las osteomusculares y los traumatismos son las principales causas de incapacidad laboral. El ausentismo es mayor en mujeres que en hombres RR 1,65 (IC95 \% 1,53-1,77). Existe también una relación directa entre el ausentismo y la edad RR 1,25 (IC95 \% 1,12-1,38).

Conclusiones La inactividad física aumenta la frecuencia y la duración de las incapacidades laborales lo cual presupone implicaciones desfavorables para el trabajador, para la empresa y para la sociedad. Los programas de promoción de la actividad física en el ámbito laboral se plantean como una opción estratégica en salud ocupacional.

Palabras Clave: Ausentismo, actividad motora (fuente: DeCS, BIREME).

\section{ABSTRACT}

Objective To study the absenteeism in regard to sex, age and physical inactivity in the work setting.

Methods Frequency, duration, cost and causes of absenteeism are studied in relation physical activity as a regular behaviour. Relative risk and its $95 \%$ confidence intervals are estimated.

Results Respiratory diseases are the first cause of sick leaves. Absenteeism is higher in women. RR 1,65 (Cl $95 \%$ 1,53-1,77), is associated to age RR 1,25 (Cl 95 \% 1,12-1,38) and to sedentariness RR 2,17 (IC $95 \%$ 1,72-2,73).

Conclusions Physical inactivity increases the rate and duration of absenteeism This fact has unfavourable implications for all: the worker, the institution and the society. 
Programs aimed at promoting physical activity in the work setting are strategic options in occupational health.

Key Words: Absenteeism, motor activity (source: MeSH, NLM).

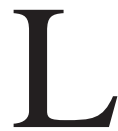

a actividad física en el ser humano y su opuesto el sedentarismo, han merecido en los últimos 50 años, la dedicación de los científicos en todo el mundo. Se ha puesto interés en averiguar sobre los riesgos que genera el ejercicio en el organismo, cuando se somete a cargas de trabajo excesivas, como ocurre ocasionalmente en el deporte competitivo y en algunas actividades laborales pesadas. De otro lado, se vienen estudiando el riesgo que representa para una persona o para una comunidad, la falta de actividades musculares vigorosas y regulares, situación ésta cada vez más frecuente, en la medida en que el desarrollo tecnológico releva a las personas de toda clase de esfuerzo físico.

El hombre moderno hace considerablemente menos esfuerzo que el hombre de otras épocas. En los años recientes se ha presentado un marcado desplazamiento de las poblaciones desde las áreas rurales hacia los centros urbanos donde la vida es excesivamente sedentaria. De otro lado, el impetuoso auge de la tecnología y la electrónica han conducido a las personas a la utilización de innumerables aparatos automáticos, capaces de realizar con lujo de eficiencia, las tareas físicas que antes eran ineludibles para el ciudadano común y corriente (1).

Estos hechos plantean algunos interrogantes lógicos: ¿Qué implica para el organismo la supresión de los estímulos fisiológicos que la actividad física produce? ¿Qué representa para los músculos y los huesos, la falta de estímulos para su fortalecimiento? ¿Qué representa para el metabolismo la disminución de activaciones de alta demanda energética? ¿Qué podrá representar para el sistema nervioso la baja utilización de neurotransmisores y de aminas neuroexitadoras? ¿Que significará para el músculo de las arterias y especialmente para el músculo del corazón la omisión de las estimulantes ráfagas de excitación? En fin, ¿Qué podrá representar para la versátil máquina humana el sometimiento de ella a prolongados períodos de desuso y pobre manutención?

En lo concerniente a la salud pública, los resultados del sedentarismo moderno no se han hecho esperar. La falta de actividad física agravada por otros hábitos nocivos del estilo de vida contemporáneo (sobrealimentación, tabaquismo, estrés, etc.) ha desatado una nueva situación epidemiológica en la cual se 
registra un marcado predominio de las enfermedades crónicas y los problemas degenerativos por atrofia y desuso $(2,3)$.

Enfermedad isquémica del corazón, enfermedad cerebro vascular, hipertensión, obesidad, osteoporosis, diabetes, ciertos tipos de cáncer, artropatías y trastornos sicológicos son todas enfermedades claramente vinculadas al sedentarismo que representan hoy en día para el mundo entero la carga más alta en morbilidad, discapacidad y muerte, y por supuesto significan también el más oneroso rubro de erogación en los presupuestos de salud $(4,5)$.

Diversos estudios epidemiológicos han demostrado el riesgo aumentado que se tiene para sufrir este tipo de enfermedades crónicas degenerativas cuando no se realiza una dosis mínima de actividad física regular. Incluso se estima que las personas inactivas tienen una vida cerca de dos años mas corta que sus contemporáneos más activos (6).

Infortunadamente las personas han disminuido dramáticamente el nivel de actividad física que realizan en sus actividades laborales y de tiempo libre; y han adoptado un estilo de vida sedentario que comparten alrededor de 60-80 por ciento de la población mundial (1).

La inactividad física se asocia a un deterioro estructural y funcional del organismo que se traduce en la aparición de diversas enfermedades, las cuales eventualmente, serian capaces de limitar las actividades productivas del ser humano en los distintos frentes de trabajo (7-9). Por todo ello, resulta pertinente estudiar el comportamiento del ausentismo laboral, describir sus características en frecuencia, duración y costo, y conocer específicamente su eventual asociación con la inactividad física.

\section{MÉTODOS}

El estudio contrasta las prevalencias de ausentismo en tres grupos humanos tomados de la misma población laboral pero que difieren en el grado de exposición a la actividad física, así:

Activos. Conformado por todos los individuos, o sea 191, que han participado regularmente y al menos por un año, en el programa para la Salud Psico-Física que ofrece el departamento de Bienestar de la Universidad de Antioquia a sus empleados. Los participantes realizan 4-5 sesiones semanales de actividad física 
moderada, cada sesión dura cerca de 60 minutos, se hace énfasis en la recreación y se complementa con conferencias sobre estilos de vida saludable.

Mixtos (Población Laboral ). Conformado por 382 sujetos seleccionados de forma aleatoria de la población laboral general, donde concurren tanto personas activas como sedentarias. Dado que este grupo representa la colectividad laboral total, lo rotularemos en lo sucesivo como Población.

Sedentarios. Constituido por 191 sujetos que se declaran sedentarios, a través de una encuesta elaborada por especialistas en el área de actividad física y salud,. El grupo proviene de la misma comunidad laboral y es comparable con los demás grupos en sus variables sociodemográficas pero difiere de ellos en relación con su apatía para la práctica de actividad física.

Para el análisis se incluyen todos los reportes de ausencia laboral o incapacidades registradas por la Empresa Promotora de Salud (EPS) de la Universidad de Antioquia, durante el periodo 1 de enero a 31 de diciembre de 2003. Se excluyeron del análisis los datos de ausentismo laboral que no tuvieran una justificación médica por enfermedad y los registros de trabajadores adscritos a otras EPS. El registro de las causas de ausentismo se realizó según la décima Clasificación Internacional de Enfermedades (CIE-10), agrupadas en los grandes grupos por sistemas orgánicos.

Se utilizó el programa SPSS versión 11.0. El análisis del ausentismo incluye la descripción y comparación de frecuencia, duración, costo y causas en la población general, según sexo y edad. Las pruebas para diferencias de promedios en muestras independientes se realizaron con base en análisis de varianza (las distribuciones F de Snedecor) y su homólogo no paramétrico Kruskall-Wallis según las diversas situaciones de cumplimiento para los supuestos de normalidad. La medida de asociación epidemiológica empleada es el riesgo relativo acompañado de su respectivo intervalo de confianza del 95 \%. Se efectuaron pruebas de significación estadística con base en la variable probabilística Chicuadrado con un nivel de significación del 5 \% para las comparaciones entre más de dos proporciones

\section{RESULTADOS}

La población afiliada a la EPS Universitaria en el 2003 está conformada por 8 337 personas, de las cuales, 4524 son beneficiarios y 3813 son cotizantes; en 
éste último grupo el 55,2 \% son hombres y el 44,8 \% mujeres, en su totalidad adultos que tienen o han tenido alguna relación laboral con la institución.

En el año 2003 se reportaron 1620 incapacidades en los cotizantes adscritos a la Universidad de Antioquia. La edad promedio de los cotizantes incapacitados fue de 48,3 años. La duración media de las ausencias fue de 6,6 días. El número de días mínimo de incapacidad fue de 1 y el máximo de 84 . El 50 \% de las incapacidades fue igual o inferior a 3 días. La frecuencia de incapacidad fue de 42,5 por cada 100 cotizantes. El costo promedio de las incapacidades fue de 377 mil pesos, con un valor mínimo de \$11 000 y un máximo de \$5 520000 .

Las seis principales causas de incapacidad en los cotizantes las constituyeron las enfermedades respiratorias 30,8 \%, las enfermedades del sistema osteomuscular $18 \%$, los traumatismos 8,8 \%, las enfermedades del sistema nervioso 8,6 \%, los trastornos mentales 6,5 \% y las enfermedades infecciosas 6,3 $\%$. Estas causas representaron el $79 \%$ del total de las ausencias ocurridas en el año 2003.

La frecuencia de incapacidad en hombres fue de 32,9 por cada 100 cotizantes y la frecuencia en mujeres fue de 54,2 en la misma referencia. El riesgo relativo es 1,65 (IC 95 \% 1,53-1,77). La duración media de las incapacidades en hombres fue de 7,2 días (IC $95 \%$ 6,79-7,60) y en las mujeres fue de 6,1 días (IC 95 $\% 5,67-6,52)$.

Se observa una relación directa entre la frecuencia de incapacidad y la edad de los empleados. La frecuencia de incapacidad varía desde 17 por 100 en los más jóvenes hasta 83 por 100 en los mayores.

Activos, Población y Sedentarios

El grupo de activos con edad promedio de 52,1 años, registró 60 incapacidades. El grupo de la población laboral con edad promedio de 51,8 tuvo 208 incapacidades. En el grupo sedentario con una edad promedio de 46,8 años, se presentaron 130 incapacidades.

La frecuencia de ausentismo en los cotizantes físicamente activos fue de 31,4 incapacidades por cada 100 personas; en los cotizantes de la población general fue de 54,5 por cada 100 y en las personas del grupo sedentario fue de 68,0 en la misma referencia. Al comparar las frecuencias del ausentismo entre 
activos y población laboral el riesgo relativo es de 1,73 (IC 95 \% 1,38-2,18). Entre activos y sedentarios el riesgo relativo es de 2,17 (IC $95 \%$ 1,72-2,73).

La comparación de las tasas de incapacidad entre activos, población laboral y sedentarios muestra una significativa tendencia a aumentar a medida que aumenta la inactividad física $\left(\mathrm{X}^{2}=53,1 \mathrm{p}=0.0\right)$ (Figura 1$)$.

Figura 1. Tasas de incapacidad según niveles de actividad física $\left(X^{2}=53,1 \quad p=.00\right)$

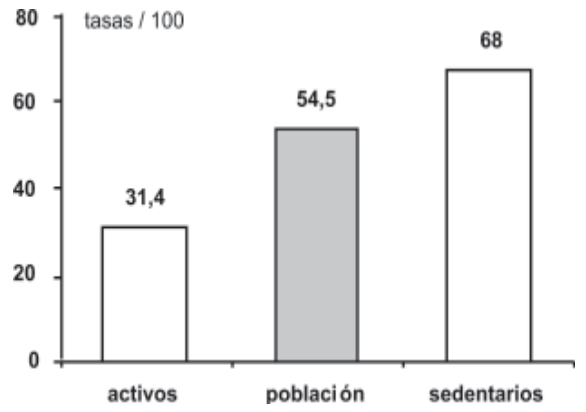

Las tasas de incapacidad por enfermedades respiratorias para activos, población laboral y sedentarios son respectivamente: 9,9, 16,8 y 23,0 por cada 100 personas $\left(\mathrm{X}^{2}=11,8 \mathrm{p}=.00\right)$. Las tasas de incapacidad por enfermedades osteomusculares para activos, población laboral y sedentarios también muestran tendencia significativa $\left(X^{2}=9,3 p=.00\right)$ y los valores respectivos son 5,2 , 11,3 y 14,7 por cada 100 personas (Figuras 2 y 3).

Figura 2. Tasas de incapacidad por enfermedad respiratoria según nivel de actividad física $\left(X^{2}=11,8 p=.00\right)$

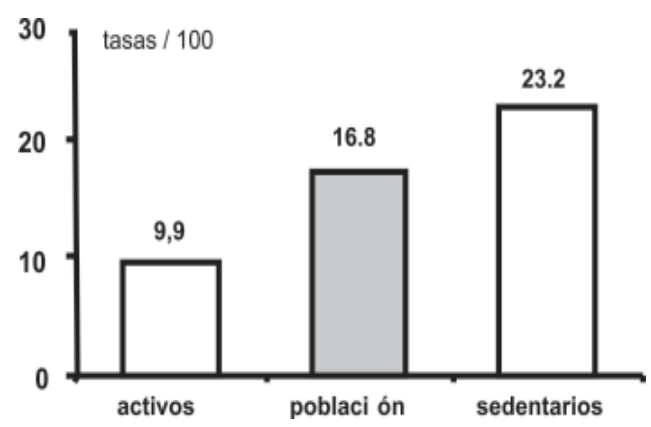


La duración media de las ausencias en los cotizantes físicamente activos es de 3,9 días (IC $95 \%$ 3,09 - 4,71), en los cotizantes de la población laboral es 5,3 días (IC $95 \%$ 4,71 - 5,89), y en los cotizantes sedentarios, la duración media de las ausencias es de 6,4 días (IC 95 \% 4,95-7,85) (Figura 4). La prueba paramétrica del análisis de varianza es significativa para esta diferencia de promedios $(\mathrm{F}=52,7 \mathrm{p}=.00)$. Dado que la distribución de frecuencia para duración de las incapacidades no sigue un patrón gausiano aplicamos la prueba no paramétrica análoga, la cual también se reporta significativa $(\mathrm{KW}=9,54 \mathrm{p}=.00)$.

Figura 3. Tasas de incapacidad por enfermedad osteomuscular según nivel de actividad física $\left(X^{2}=9,3 \quad p=.00\right)$

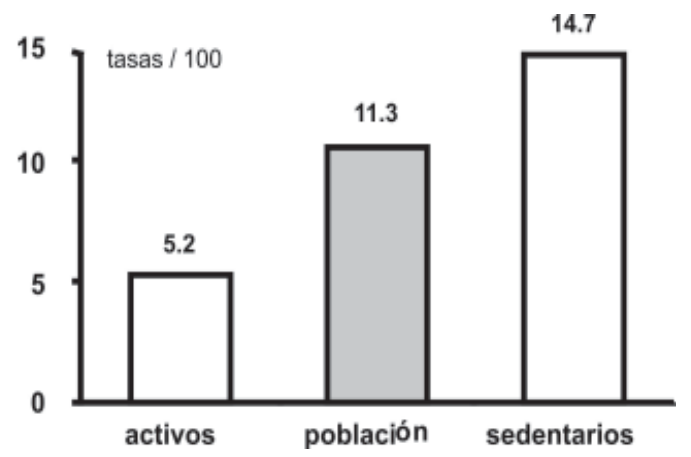

Figura 4. Promedio en días de duración por incapacidad según nivel de actividad física ( $\mathrm{KW}=9, .5 \mathrm{p}=.00$ )

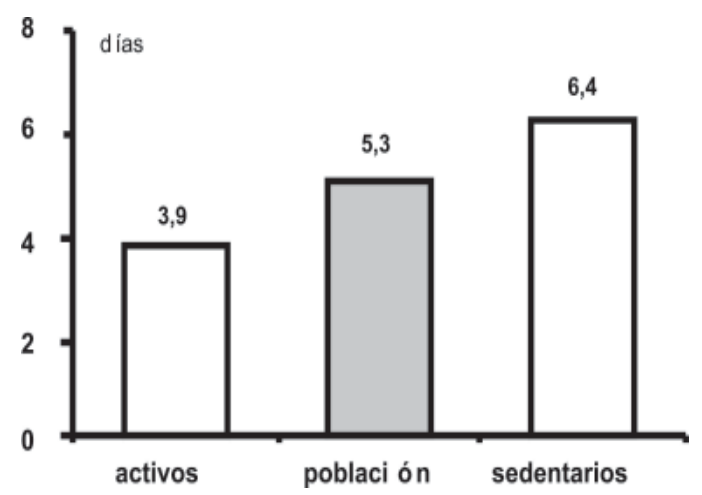

Una persona activa se incapacita en promedio 1,23 días al año, una persona de la población laboral lo hace 2,8 días al año y una persona sedentaria 4,35 días/año. 
El costo total de las 1620 incapacidades en los 3813 cotizantes durante el año asciende a 610,7 millones de pesos, es decir unos 1700 SMMV (salarios mínimos mensuales vigentes para el año 2003), lo cual representa un costo promedio de 377000 pesos (US\$ 190) por incapacidad.

El total de días de incapacidad que se presentaron en la comunidad laboral fue de 10660 días. Si toda la comunidad fuera físicamente activa, los días de incapacidad estimados se reducirían a 4 690. Si en cambio toda la comunidad laboral fuera sedentaria, los días de incapacidad se elevarían a 16587.

$\mathrm{Al}$ aceptar que la actividad física permitiría ahorrar 5970 días de incapacidad por año en la comunidad laboral y con un costo por día de 57300 pesos, la cantidad de dinero ahorrado por la institución ascendería a 342,1 millones de pesos, lo cual implicaría una reducción de 56 \% en la factura anual por incapacidades laborales.

\section{DISCUSIÓN}

Las principales causas de incapacidad en la población laboral son las enfermedades respiratorias, las osteomusculares y los traumatismos. Estos hallazgos concuerdan con los resultados reportados en otros estudios y de hecho coinciden con las causas comunes de morbilidad en la población general, lo cual a su vez sugiere que los determinantes de enfermedad general no dependen tanto de factores laborales como extralaborales y que afectan a los seres humanos siempre que ocurra una exposición suficiente a los factores de riesgo ambientales y de comportamiento $(2,3)$.

En el análisis según sexo se encuentra que las mujeres se ausentan del trabajo con más frecuencia que los hombres. Este hecho ha sido reportado en la literatura científica y reconoce que las mujeres concurren con mayor frecuencia a los servicios asistenciales y se prodigan mayores cuidados contra diversos factores de riesgo. Tal situación, eventualmente contraproducente en el desempeño laboral de las mujeres, les confiere ventajas en aspectos como la esperanza de vida donde superan, por lo general, en 5-10 años la sobrevida de los hombres (10).

La edad es sin duda un factor determinante en la frecuencia de las incapacidades médicas. Por cada día de incapacidad en los cotizantes de menor edad se presentan 1,6 días de incapacidad en los cotizantes de mayor edad 
(mayores al percentil 50), fenómeno que puede estar explicado por los procesos de deterioro funcional y orgánico característicos del envejecimiento y el efecto agregado de los años de trabajo. No obstante, la actividad física pudiera actuar como un factor protector, pues en el presente estudio los activos presentan bajas tasas de incapacidad a pesar de ostentar los mayores promedios de edad (7).

En nuestro estudio, la frecuencia de incapacidad en los sedentarios excede en un $25 \%$ la frecuencia de incapacidad en la población laboral y en $117 \%$ la de las personas activas. La mayor incidencia de incapacidad laboral ha sido confirmada con diversas magnitudes y en múltiples estudios, algunos de los cuales han hecho seguimiento de grupos poblacionales numerosos en distintas regiones del mundo $(8,9)$.

La menor frecuencia de ausentismo por enfermedades osteomusculares en los activos, de alguna manera es un resultado que muestra los beneficios preventivos de la implementación de un programa de ejercicio físico orientado hacia la salud y no a la competencia deportiva. Ocasionalmente se ha reportado un aumento en la frecuencia de lesiones músculo-esqueléticas, relacionadas con deportes competitivos y ejercicios muy intensos. Los programas que promueven la competencia entre equipos empresariales y en deportes que implican contacto físico arriesgan la ocurrencia de acciones violentas y pudieran ser contraproducentes para el bienestar laboral. Es claro que los programas de actividad física para la promoción de la salud en las empresas se deben pensar e implementar sobre la base de actividades deportivas de tipo recreativo y formativo, que se realizan a intensidad moderada y con espíritu de amistad y compañerismo (8).

Los resultados señalan que la población sedentaria no solo se incapacita con mayor frecuencia, sino además que la duración de la incapacidad es significativamente mayor con relación a los cotizantes físicamente activos. Esto podría sugerir que la recuperación es mejor y es más rápida en las personas físicamente activas, lo cual también ha sido ya reportado en la literatura mundial (7-9).

El total de días de incapacidad, producto de la frecuencia por la duración, nos permite concluir que por cada día de incapacidad que se genera en un individuo activo, se presentan 2-3 en la comunidad laboral general y 3-4 días de incapacidad en los sedentarios. 
Es preciso admitir que el ausentismo no es sólo indicador de enfermedad, sino también de insatisfacción en el trabajo y que pueden ser importantes otros factores explicativos no considerados en el estudio, como la percepción del empleado sobre su trabajo, las relaciones laborales, la remuneración y las tareas rutinarias, etc. Se ha establecido que los trabajadores sedentarios reportan mayor cantidad de disfunciones sicológicas que quienes tienen hábitos de vida activa y practican deportes (11-13).

Como factores explicativos de la asociación encontrada se puede considerar también el aporte del ejercicio moderado en la función inmunitaria de hombres y mujeres, aspecto que contribuye a explicar en cierta medida la menor frecuencia de incapacidad por causas médicas presentada en el grupo de personas activas (14).

Los importantes beneficios de la práctica regular de actividad física sobre la salud mental podrían jugar un papel decisivo en los niveles de satisfacción personal y laboral. La participación en un programa de mantenimiento físico proporciona una alternativa para salir de la rutina y la presión del trabajo. La actividad física recreativa reduce el estrés y el riesgo de presentar trastornos psicológicos como ansiedad y depresión, los cuales en la actualidad se reconocen como factores relacionados con padecimientos agudos y crónicos en poblaciones laboralmente activas (15-17).

El ejercicio físico está asociado con un mejor estado de salud y con bajos niveles de riesgo para las principales causas de morbilidad y mortalidad por enfermedades crónicas. Estudios epidemiológicos han demostrado su efecto preventivo en diversas patologías como las enfermedades cardiovasculares, el infarto agudo de miocardio, la diabetes, el cáncer, la osteoporosis, la obesidad y la depresión (18-21). Un programa regular de actividad física, moderado en intensidad, diversificado, recreativo y pensado para el disfrute y no para la competencia obsesiva podría ser una poderosa herramienta de bienestar laboral y salud ocupacional al alcance de prácticamente todas las empresas (2225). La inactividad física aumenta la frecuencia, la duración y los costos de las incapacidades laborales, lo cual conlleva implicaciones desfavorables para todos. Para el trabajador, pues es quien sufre la enfermedad, para la empresa que por su parte, pierde en productividad y para la sociedad en general que ve retrasado su crecimiento económico y social.

Estos hechos deberían tenerse muy en cuenta para la implementación de programas de promoción de la salud, incluso en el ámbito mismo de la empre- 
sa, pues las ganancias podrían compensar generosamente los recursos y los esfuerzos en ellos invertidos •

\section{REFERENCIAS}

1. World Health Organization. Sedentary lifestyle a global public health problem. Geneva: World Health Organization; 2002.

2. Taylor P. Some international trends in sickness medical. British Medical Journal. 1999; 12:195058.

3. Franson E, Ahlbom A, Reuterwall C, Hallqvist J, Alfredsson L. The risk of acute miocardial infarction: Interactions of types of physical activity. International Journal of Epidemiology. 2004; 15(5):573-582.

4. Friedenreich $\mathrm{CM}$, Orenstein MR. Physical activity and cancer prevention: Etiologic evidence and biological mechanisms. Nutrition. 2002; 132:345-364.

5. Stromme SB, Hostmark AT. Physical activity overweight and obesity. Sports Medicine. 2000; 120(2):578-582.

6. Blair SN, Kohl HW, Barlow CE, Paffenbarger RS, Gibbons LW, Macera CA. Changes in physical fitness and all-cause mortality. A prospective study of healthy and unhealthy men. JAMA. 1995; 273:1093-1098.

7. Pelletier B, Boles M, Lynch W. Change in health risks and work productivity over time. Journal of Occupational and Environmental Medicine. 2004; 46(7):746-754.

8. Van Amelvoort LG, Spigt MG, Swean GM, Kant I. Leisure time physical activity and sickness absenteeism: a prospective study. Occup Med. 2006; 56(3):210-22.

9. Muto T, Sakurai H. Relation between exercise and absenteeism due to illness and injury in manufacturing companies in Japan. J Occup Med. 1993; 35(10): 995-9.

10. Steinhart M, Greehow L, Stewart J. The relationship of physical activity and cardiovascular fitness to absenteeism and medical care claims among law enforcement officer. Am J Health Promot. 1991; 5(6): 455-60.

11. Fox KR. The influence of physical activity on mental well-being. Public Health Nutrition. 1999; 2(3):411-418.

12. Weyerer S. Physical inactivity and depression in the community: Evidence from the upper Bavarian Field Study. International J Sports Medicine. 1992; 13:492-496.

13. Bernaards CM, Jans MP, Van den Heuvel SG, Hendriksen IJ, Houtman IL, Bongers PM. Can strenuous leisure time physical activity prevent psychological complaints in a working population? Occup Environ. Med. 2006; 63:10-16.

14. Fairey AS, Courneys KS, Field CJ, Mackey JR. Physical exercise and immune system function in cancer survivors: A comprehensive review and future directions. Cancer. 2002; 94:539-551.

15. Blair S, Cheng $\mathrm{Y}$, Holder J. Is physical activity or physical fitness more important in defining health benefits. Medicine Science and Sports Exercise. 2001; 33(6):379-399.

16. Baun WB, Bernacki EJ, Tsai SP. A preliminary investigation: Effect of a corporate fitness program on absenteeism and health care cost. Journal of Occupational Medicine. 1986; 28(1):18-22.

17. Bell BC, Blanke DJ. The effects of an employee fitness program on health care costs and utilization. Health Values. 1992:16(3):3-13.

18. WHO. Centers for Disease and Prevention Control. Physical activity policy development: A synopsis of the WHO/CDC consultation. Atlanta, GA: WHO. Centers for disease and prevention control; 2002.

19. Donatele RJ, Hawkins MJ. Employee stress claims: Increasing implications for health promotion programming. American J of Health Promotion. 1999; 3:19-25. 
20. Wannamethee SG, Shaper AG. Physical activity in the prevention of cardiovascular disease: An epidemiological perspective. Sports Medicine. 2001; 34(2):101-114.

21. Nichols DL, Sanborn CF, Bonnick SL, Ben Ezra V, Gench B, DiMarco NM. The effects of gymnastics training on bone mineral density. Medicine Science and Sports Exercise. 1994; 26:1220-1225.

22. Pratt M, Jacoby E. Promoting physical activity in the Americas. Medicine Science and Sports Exercise. 2004; 25(2):183-190.

23. Tai SS, Gould MA, Smith PL, Lliffe SE. Promoting physical activity in general practice: Should prescribed exercise be free? Journal of the Royal Society of Medicine. 1999; 92(2):6587.

24. Proper KI, Koning M, van der Beek AJ, Hildebrandt VH, Bosscher RJ, Van Mechelen W. The effectiveness of worksite physical activity programs on physical activity, physical fitness and health. Clin J Sport Med. 2003 Mar; 13(2):106-17.

25. Van den Heuvel S G, Boshuizen H C, Hildebrandt V H, Blatter B M, Ariëns G A, Bongers P M. Effect of sporting activity on absenteeism in a working population. Br J Sports Med. 2005; 39:1-5. 